\title{
Integrable lambda models and Chern-Simons theories
}

\section{David M. Schmidtt}

Departamento de Física, Universidade Federal de São Carlos, Caixa Postal 676, CEP 13565-905, São Carlos-SP, Brazil

E-mail: david@df.ufscar.br

ABSTRACT: In this note we reveal a connection between the phase space of lambda models on $S^{1} \times \mathbb{R}$ and the phase space of double Chern-Simons theories on $D \times \mathbb{R}$ and explain in the process the origin of the non-ultralocality of the Maillet bracket, which emerges as a boundary algebra. In particular, this means that the (classical) $A d S_{5} \times S^{5}$ lambda model can be understood as a double Chern-Simons theory defined on the Lie superalgebra $\mathfrak{p} \mathfrak{s u}(2,2 \mid 4)$ after a proper dependence of the spectral parameter is introduced. This offers a possibility for avoiding the use of the problematic non-ultralocal Poisson algebras that preclude the introduction of lattice regularizations and the application of the QISM to string sigma models. The utility of the equivalence at the quantum level is, however, still to be explored.

Keywords: Chern-Simons Theories, Integrable Field Theories, Sigma Models, Integrable Hierarchies

ArXiv EPrint: 1701.04138 


\section{Contents}

1 Introduction 1

2 Integrable lambda models $\quad 3$

3 Double Chern-Simons theory $\quad 6$

4 Concluding remarks $\quad 16$

\section{Introduction}

It is by now widely recognized that integrability plays a fundamental role on the AdS/CFT correspondence and that a way to explore the duality more efficiently is to study its underlying integrable structure in a systematic way. One logical strategy to do so is to implement deformations in a consistent mathematical way and then learn more about the physical system from its response to the deformation. Recently, two different but complementary kinds of deformations defined on the gravity side of the duality have been introduced $[4,7]$. Both preserve the integrability of (super)-string sigma models and are currently known as the eta models $[1,3-5]$ and the lambda models $[2,6-8]$. The works [3-5] and [6-8], respectively, came as generalizations of the original ideas for deforming sigma models introduced by Klimčík in [1] for the eta models and by Sfetsos in [2] for the lambda models. In the particular case of the $A d S_{5} \times S^{5}$ Green-Schwarz (GS) superstring, the main property is that its eta/lambda model realize a quantum group deformation of their parent sigma model S-matrix with a $q$ that is real and a root-of-unity [9-11], respectively.

Most of the physically interesting integrable field theories (including the ones mentioned above) are of the so-called non-ultralocal type, a property that poses a major obstacle to the use of powerful techniques like the algebraic Bethe ansatz and this is why a great amount effort has been invested along the years in trying to eliminate this "pathological" behavior e.g. see [12-19] for several different approaches concerning this issue. The most important work dealing successfully with this problem is the 1986 seminal paper by Faddeev and Reshetikhin (FR) [12], in which a (rather ad hoc) ultralocalization method for the $\mathrm{SU}(2)$ principal chiral model (PCM) was introduced allowing to exactly quantize the theory within the QISM scheme. Unfortunately, the method only seemed to work with this case and not with the more interesting PCM's on any Lie group $G$ or the more general sigma models on symmetric spaces $F / G$. It was only in 2012 where real progress was made by Delduc, Magro and Vicedo [18], in which the underlying algebraic mechanism behind the ultralocalization method of FR was discovered, generalized and applied to any PCM 
and sigma model on (semi)-symmetric spaces. ${ }^{1}$ Unfortunately, in the case of sigma models on (semi)-symmetric spaces the non ultralocality is still present albeit in an alleviated way and the introduction of a lattice regularization (at quantum level) for the alleviated theories is still not known because of the non-ultralocality persists. ${ }^{2}$

One of the main characteristics of the lambda deformation is that it implements the FR mechanism of [18] directly at the Lagrangian level [24] and this is the best we can do (to present knowledge) in handling analytically the non ultra-locality of the integrable field theory from a world-sheet theory point of view. This means, in particular, that the problem is still present so apparently nothing seems to be gained by deforming the original theory in this particular way. However, it is the same deformed theory that suggests there is a way out if we give up the world-sheet description.

In this work we offer a new approach to deal with the non ultralocality of all known lambda models, which have recently attracted a lot of attention. The idea is not to tackle the problem in $1+1$ dimensions, as customary, but rather from a $2+1$ dimensional point of view. As we shall see, by changing the dimensionality the problem ceases to exist (for any value of the deformation parameter $\lambda$ ) and the strategy to do it is to exploit the natural relationship that exist between WZW models and Chern-Simons (CS) theories. We are also able to introduce the spectral parameter in the $2+1$ theory giving it a more prominent role. We expect this approach will provide a novel way to treat the $1+1$ integrable field theories that fit within the formulation of lambda models but one of the hopes is to leave open the possibility of generalizing the construction so that more general theories can be treated in a similar way. For a new but different approach to non-ultralocal integrable field theories, see the very recent work [22]. See also [23] for another recent application of the QISM to the lambda model of the PCM.

The lambda models have two important characteristic properties that are analogues of similar relations present on ordinary chiral WZW models. They are summarized in the following pair of (on-shell) results [24, 25]

$$
m\left(z_{ \pm}\right)=P \exp \left[ \pm \frac{2 \pi}{k} \int_{S^{1}} d \sigma \mathscr{J}_{\mp}(\sigma)\right] \text { and } \mathcal{F}=\Psi\left(z_{+}\right) \Psi\left(z_{-}\right)^{-1} .
$$

In the first equation, $m(z)$ is the monodromy matrix of the $2 \mathrm{~d}$ theory, $z_{ \pm}=\lambda^{ \pm 1 / 2} \in \mathbb{R}$ are two special values of the spectral parameter $z$ and $\mathscr{J}_{ \pm}$are two currents satisfying the algebra of two mutually commuting Kac-Moody algebras. This relation have been studied in $^{3}$ [24] for bosonic sigma models and after the use of a KM lattice regularization results in the presence of a quantum group symmetry with a deformation parameter $q$ that is a root of unity.

In the second equation, we have that $\mathcal{F}$ is the Lagrangian matrix field entering the definition of the lambda model action and $\Psi(z)$ is the wave function that appears as the compatibility equation for the Lax pair representation of the equations of motion [25,

\footnotetext{
${ }^{1}$ The $A d S_{5} \times S^{5}$ superstring was considered in [20, 21].

${ }^{2}$ To the present, it has been only possible to construct a lattice Poisson algebra that is related to the Pohlmeyer reduction of the string sigma models [18, 20, 21].

${ }^{3}$ This paper is strongly inspired by the results of $[26]$.
} 
41]. A similar decomposition appears for ordinary chiral WZW models but with the very important difference that for the lambda models the elements $\Psi\left(z_{ \pm}\right)$are far from being chiral. ${ }^{4}$ As it is well known [44-46], conventional WZW models are deeply related to 3d Chern-Simons gauge theories and under this connection, the non ultralocality of the KacMoody chiral algebras of the WZW model rises as a boundary effect after the impositions of certain constraints on the phase space. We will see below that this situation persist also for lambda models but with the added advantage that a spectral parameter can be naturally introduced and that this time it is the Maillet bracket [43] that emerges as boundary algebra. Hopefully, this remarkable relation will reveal unexpected connections between integrable string sigma models and gauge theories of the CS type that might assist in the quantization of the former theories.

The paper is organized as follows. In section 1, we introduce the lambda models and emphasize the properties that are important for the topic of the present study. In section 2, we elaborate on the version of the Chern-Simon theory that, after introduction of the spectral parameter, turns out to be equivalent to the lambda models at the classical level. We finish with some remarks concerning our approach and mention on problems to be considered in the near future.

\section{Integrable lambda models}

In this section we briefly review the most important aspects of the integrable deformations that are of relevance for the present paper. We will restrict the discussion to the specific example of the lambda model of the Green-Schwarz (GS) superstring on the coset superspace $A d S_{5} \times S^{5}$ but also make contact with similar lambda models when useful for clarifying purposes.

Consider the Lie superalgebra $\mathfrak{f}=\mathfrak{p s u}(2,2 \mid 4)$ of $F=P \mathrm{SU}(2,2, \mid 4)$ and its $\mathbb{Z}_{4}$ decomposition induced by the automorphism $\Phi$

$$
\Phi\left(\mathfrak{f}^{(m)}\right)=i^{m} \mathfrak{f}^{(m)}, \quad \mathfrak{f}=\bigoplus_{i=0}^{3} \mathfrak{f}^{(i)}, \quad\left[\mathfrak{f}^{(m)}, \mathfrak{f}^{(n)}\right] \subset \mathfrak{f}^{(m+n) \bmod 4},
$$

where $m, n=0,1,2,3$. From this decomposition we associate the following twisted loop superagebra

$$
\widehat{\mathfrak{f}}=\bigoplus_{n \in \mathbb{Z}}\left(\bigoplus_{i=0}^{3} \mathfrak{f}^{(i)} \otimes z^{4 n+i}\right)=\bigoplus_{n \in \mathbb{Z}} \widehat{\mathfrak{f}}^{(n)}
$$

which is required to exhibit the integrable properties of the theory in terms of the spectral parameter $z$. Denote by $G$ the bosonic Lie group associated to $\mathfrak{f}^{(0)}=\mathfrak{s u}(2,2) \times \mathfrak{s u}(4)$.

The lambda model on the semi-symmetric space $F / G$ is defined by the following action functional ${ }^{5}[7]$

$$
S=S_{F / F}\left(\mathcal{F}, A_{\mu}\right)-\frac{k}{\pi} \int_{\Sigma} d^{2} \sigma\left\langle A_{+}(\Omega-1) A_{-}\right\rangle, \quad k \in \mathbb{Z},
$$

\footnotetext{
${ }^{4}$ Precisely, this decomposition is used in [50] to construct the deformed giant magnon solutions of lambda models.

${ }^{5}$ The $1+1$ notation used in this paper is: $\sigma^{ \pm}=\tau \pm \sigma, \partial_{ \pm}=\frac{1}{2}\left(\partial_{\tau} \pm \partial_{\sigma}\right), \eta_{\mu \nu}=\operatorname{diag}(1,-1), \epsilon^{01}=1$, $\delta_{\sigma \sigma^{\prime}}=\delta\left(\sigma-\sigma^{\prime}\right)$ and $\delta_{\sigma \sigma^{\prime}}^{\prime}=\partial_{\sigma} \delta\left(\sigma-\sigma^{\prime}\right)$. We also have that $a_{ \pm}=\frac{1}{2}\left(a_{\tau} \pm a_{\sigma}\right)$.
} 
where $\langle *, *\rangle=\operatorname{STr}(*, *)$ is the supertrace in some faithful representation of the Lie superalgebra $\mathfrak{f}, \Sigma=S^{1} \times \mathbb{R}$ is the world-sheet manifold parameterized by $(\sigma, \tau)$ and $\Omega \equiv \Omega(\lambda)$, where

$$
\Omega(z)=P^{(0)}+z P^{(1)}+z^{-2} P^{(2)}+z^{-1} P^{(3)}
$$

is the omega projector characteristic of the GS superstring. The $P^{(m)}$ are projectors along the graded components $\mathfrak{f}^{(m)}$ of $\mathfrak{f}$. Above, we have that

$S_{F / F}\left(\mathcal{F}, A_{\mu}\right)=S_{W Z W}(\mathcal{F})-\frac{k}{\pi} \int_{\Sigma} d^{2} \sigma\left\langle A_{+} \partial_{-} \mathcal{F} \mathcal{F}^{-1}-A_{-} \mathcal{F}^{-1} \partial_{+} \mathcal{F}-A_{+} \mathcal{F} A_{-} \mathcal{F}^{-1}+A_{+} A_{-}\right\rangle$,

where $S_{W Z W}(\mathcal{F})$ is the usual level $k$ WZW model action. The original GS superstring coupling constant is ${ }^{6} \kappa^{2}$ and it is related to $k$ through the relation $\lambda^{-2}=1+\kappa^{2} / k$. From (2.3) we realize that the $\lambda$-deformation can be seen as a continuation of the GS superstring into a topological field theory defined by the gauged $F / F$ WZW model.

The gauge field equations of motion are given by

$$
A_{+}=\left(\Omega^{T}-D^{T}\right)^{-1} \mathcal{F}^{-1} \partial_{+} \mathcal{F}, \quad A_{-}=-(\Omega-D)^{-1} \partial_{-} \mathcal{F} \mathcal{F}^{-1}, \quad D=A d_{\mathcal{F}} .
$$

After putting them back into the action (2.3), a deformation of the non-Abelian T-dual of the GS superstring with respect to the global left action of the supergroup $F$ is produced. A dilaton is generated in the process but we will not consider its effects here as we are only concerned with the classical aspects of the theory.

The $\mathcal{F}$ equations of motion, when combined with (2.6) can be written in two different by equivalent ways

$$
\left[\partial_{+}+\mathscr{L}_{+}\left(z_{ \pm}\right), \partial_{-}+\mathscr{L}_{-}\left(z_{ \pm}\right)\right]=0
$$

where

$$
\mathscr{L}_{ \pm}(z)=I_{ \pm}^{(0)}+z I_{ \pm}^{(1)}+z^{ \pm 2} I_{ \pm}^{(2)}+z^{-1} I_{ \pm}^{(3)}
$$

is the GS superstring Lax pair that besides satisfy the condition

$$
\Phi\left(\mathscr{L}_{ \pm}(z)\right)=\mathscr{L}_{ \pm}(i z)
$$

Then, the lambda model equations of motion follow from zero curvature condition of $\mathscr{L}_{ \pm}(z)$. Above, the $I_{ \pm}^{(m)}$, are the components of the deformed dual currents defined by

$$
I_{+}=\Omega^{T}\left(z_{+}\right) A_{+}, \quad I_{-}=\Omega^{-1}\left(z_{-}\right) A_{-}, \quad z_{ \pm}=\lambda^{ \pm 1 / 2} .
$$

The flatness of the Lax pair is equivalent to the compatibility condition

$$
\left(\partial_{\mu}+\mathscr{L}_{\mu}(z)\right) \Psi(z)=0
$$

where $\Psi(z)$ is the so-called wave function. This last equation together with (2.6) and (2.8) allow to relate (on-shell) the Lagrangian fields of the lambda model to the wave function [25, 41]. For example,

$$
\mathcal{F}=\Psi\left(z_{+}\right) \Psi\left(z_{-}\right)^{-1}, \quad A_{ \pm}=-\partial_{ \pm} \Psi\left(z_{ \pm}\right) \Psi\left(z_{ \pm}\right)^{-1} .
$$

\footnotetext{
${ }^{6}$ To match with the notation of $[25,50]$, take $\kappa^{2}=4 \pi g$.
} 
The spatial component of the Lax pair $\mathscr{L}_{\sigma}(z) \equiv \mathscr{L}(z)$ satisfy

$$
\mathscr{L}\left(z_{ \pm}\right)=\mp \frac{2 \pi}{k} \mathscr{J}_{\mp},
$$

where the currents $\mathscr{J}_{ \pm}$obey the relations of two mutually commuting Kac-Moody algebras ${ }^{7}$

$$
\left\{\stackrel{1}{\mathscr{J}}_{ \pm}(\sigma), \stackrel{2}{\mathscr{J}}_{ \pm}\left(\sigma^{\prime}\right)\right\}=-\left[C_{12}, \stackrel{2}{\mathscr{J}}_{ \pm}\left(\sigma^{\prime}\right)\right] \delta_{\sigma \sigma^{\prime}} \mp \frac{k}{2 \pi} C_{12} \delta_{\sigma \sigma^{\prime}}^{\prime}
$$

Equation (2.13) is valid for all lambda models and as a consequence of this the first relation in (1.1) provide conserved Lie-Poisson charges [24]. On the constrained surface defined by (2.6) the KM currents take the form

$$
\mathscr{J}_{+}=\frac{k}{2 \pi}\left(\Omega^{T} A_{+}-A_{-}\right), \quad \mathscr{J}_{-}=-\frac{k}{2 \pi}\left(A_{+}-\Omega A_{-}\right)
$$

and are used to relate $\mathscr{J}_{ \pm}$with the deformed dual currents $I_{ \pm}$. This is a particularly useful relation because it means the current algebra for $I_{ \pm}$follows from the algebra (2.14).

By adding to the Lax operator arbitrary $z$-dependent terms proportional to the Hamiltonian constraints (bosonic and fermionic) of the theory and by demanding that the condition (2.9) and the equation (2.13) are still valid, we obtain the Hamiltonian or extended Lax operator $[8,25,41]$

$$
\begin{aligned}
\mathscr{L}^{\prime}(z)= & -\frac{2 \pi}{k} \frac{\left(z^{4}-z_{+}^{4}\right)}{\left(z_{+}^{4}-z_{-}^{4}\right)}\left\{\mathscr{J}_{+}^{(0)}+\frac{z_{-}^{3}}{z^{3}} \mathscr{J}_{+}^{(1)}+\frac{z_{-}^{2}}{z^{2}} \mathscr{J}_{+}^{(2)}+\frac{z_{-}}{z} \mathscr{J}_{+}^{(3)}\right\} \\
& -\frac{2 \pi}{k} \frac{\left(z^{4}-z_{-}^{4}\right)}{\left(z_{+}^{4}-z_{-}^{4}\right)}\left\{\mathscr{J}_{-}^{(0)}+\frac{z_{+}^{3}}{z^{3}} \mathscr{J}_{-}^{(1)}+\frac{z_{+}^{2}}{z^{2}} \mathscr{J}_{-}^{(2)}+\frac{z_{+}}{z} \mathscr{J}_{-}^{(3)}\right\} .
\end{aligned}
$$

Then, as a consequence of the Kac-Moody algebra structure of the theory (2.14), the Hamiltonian Lax operator obeys the Maillet algebra

$$
\begin{aligned}
\left\{\mathscr{L}^{\prime}(\sigma, z), \mathscr{L}^{\prime}\left(\sigma^{\prime}, w\right)\right\}= & -\left[\mathfrak{r}_{12}, \mathscr{L}^{\prime}(\sigma, z)+\stackrel{\mathscr{L}}{ }^{\prime}\left(\sigma^{\prime}, w\right)\right] \delta_{\sigma \sigma^{\prime}} \\
& +\left[\mathfrak{s}_{12}, \mathscr{L}^{\prime}(\sigma, z)-\stackrel{\mathscr{L}}{ }^{\prime}\left(\sigma^{\prime}, w\right)\right] \delta_{\sigma \sigma^{\prime}}-2 \mathfrak{s}_{12} \delta_{\sigma \sigma^{\prime}}^{\prime}
\end{aligned}
$$

which reduce to the two mutually commuting Kac-Moody algebras at the special points $z_{ \pm}$. We will deduce this bracket from a Chern-Simons theory point of view and write down the explicit form of the $\mathfrak{r} / \mathfrak{s}$ operators below. It is important to mention that both GS and hybrid superstring formulations share the same extended Lax operator [8] but defined in terms of the Lie superalgebras $\mathfrak{p s u}(2,2 \mid 4)$ and $\mathfrak{p s u}(1,1 \mid 2)$, respectively.

The last piece of information is related to the imposition of the Virasoro constraints $T_{ \pm \pm} \approx 0$, which renders the lambda model a string theory. ${ }^{8}$ The stress-tensor components

\footnotetext{
${ }^{7}$ The Kac-Moody algebras are protected and does not change under the Dirac procedure [6] meaning we can use them on the constrained surface defined by (2.6).

${ }^{8}$ The lambda models are also consistent superstring theories at the quantum level, as has been recently shown in [51-53] for $A d S_{n} \times S^{n}, n=2,3,5$.
} 
of the action (2.3) are given by

$$
T_{ \pm \pm}=-\frac{k}{4 \pi}\left\langle\left(\mathcal{F}^{-1} D_{ \pm} \mathcal{F}\right)^{2}+2 A_{ \pm}(\Omega-1) A_{ \pm}\right\rangle
$$

where $D_{ \pm}(*)=\partial_{ \pm}(*)+\left[A_{ \pm}, *\right]$. On the surface defined by the gauge field equations of motion they reduce to the usual quadratic form albeit in terms of the deformed dual currents

$$
T_{ \pm \pm}=\frac{k}{4 \pi}\left(z_{+}^{4}-z_{-}^{4}\right)\left\langle I_{ \pm}^{(2)} I_{ \pm}^{(2)}\right\rangle,
$$

that in terms of the Lax pair become

$$
T_{ \pm \pm}= \pm \frac{k}{4 \pi}\left\langle\mathscr{L}_{ \pm}^{2}\left(z_{+}\right)-\mathscr{L}_{ \pm}^{2}\left(z_{-}\right)\right\rangle
$$

From this last expression we can extract the Hamiltonian and momentum densities ${ }^{9}$

$$
\begin{aligned}
H & =\frac{k}{4 \pi}\left\langle\mathscr{L}_{\tau}\left(z_{+}\right) \mathscr{L}_{\sigma}\left(z_{+}\right)-\mathscr{L}_{\tau}\left(z_{-}\right) \mathscr{L}_{\sigma}\left(z_{-}\right)\right\rangle, \\
P & =\frac{k}{8 \pi}\left\langle\left(\mathscr{L}_{\tau}^{2}\left(z_{+}\right)+\mathscr{L}_{\sigma}^{2}\left(z_{+}\right)\right)-\left(\mathscr{L}_{\tau}^{2}\left(z_{-}\right)+\mathscr{L}_{\sigma}^{2}\left(z_{-}\right)\right)\right\rangle .
\end{aligned}
$$

The expression (2.20) is not unique to the GS superstring and could be considered as a starting point. Indeed, if we take for example the Lax pair for the hybrid superstring on $A d S_{2} \times S^{2}$ given by [8]

$$
\mathscr{L}_{+}(z)=I_{+}^{(0)}+z I_{+}^{(1)}+z^{2} I_{+}^{(2)}+z^{3} I_{+}^{(3)}, \quad \mathscr{L}_{-}(z)=I_{-}^{(0)}+z^{-3} I_{-}^{(1)}+z^{-2} I_{-}^{(2)}+z^{-1} I_{-}^{(3)},
$$

which also satisfy (2.9) and make use of (2.20), we do recover the known expressions for the stress-tensor

$$
T_{ \pm \pm}=\frac{k}{4 \pi}\left(z_{+}^{4}-z_{-}^{4}\right)\left\langle I_{ \pm}^{(2)} I_{ \pm}^{(2)}+2 I_{ \pm}^{(1)} I_{ \pm}^{(3)}\right\rangle
$$

but in terms of a different set of deformed dual currents $I_{ \pm}$written down in [8]. This result also applies to the PCM lambda model but with a different set of points $z_{ \pm}$defined in [24].

As we saw above, the lambda models are naturally equipped with two decoupled Kac-moody algebras and the Lagrangian field decompose in a rather similar way as the Lagrangian field in conventional chiral WZW models. This suggest that the known [44-46] relation between WZW models and CS theories could be present for lambda models as well and we now proceed to make this connection more precise.

\section{Double Chern-Simons theory}

Consider the following double Chern-Simons action functional defined by ${ }^{10}$

$$
S_{C S}=S_{(+)}+S_{(-)},
$$

\footnotetext{
${ }^{9}$ Use $H=T_{++}+T_{--}$and $P=T_{++}-T_{--}$.

${ }^{10} \mathrm{We}$ do not know if there is a standard name in the literature for this type of action.
} 
where

$$
S_{( \pm)}= \pm \frac{k}{4 \pi} \int_{M}\left\langle\mathcal{A}_{( \pm)} \wedge \hat{d} \mathcal{A}_{( \pm)}+\frac{2}{3} \mathcal{A}_{( \pm)} \wedge \mathcal{A}_{( \pm)} \wedge \mathcal{A}_{( \pm)}\right\rangle .
$$

The $( \pm)$ sub-index is just a label whose significance will emerge later on, $M$ is a 3 dimensional manifold and $\mathcal{A}_{( \pm)}$are two different 3-dimensional gauge fields valued in the Lie superalgebra $\mathfrak{f}$. In what follows we will study the generic action

$$
S=\frac{\bar{k}}{4 \pi} \int_{M}\left\langle\mathcal{A} \wedge \hat{d} \mathcal{A}+\frac{2}{3} \mathcal{A} \wedge \mathcal{A} \wedge \mathcal{A}\right\rangle, \quad \bar{k}= \pm k \quad \text { for } \quad( \pm)
$$

to avoid a duplicated analysis.

In order to define the Hamiltonian theory of our interest we consider the action on the manifold $M=D \times \mathbb{R}$, where $D$ is a 2 -dimensional disc parameterized by $x^{i}, i=1,2$ and $\mathbb{R}$ is the time direction parameterized by $\tau$. It is useful to use radius-angle coordinates $(r, \sigma)$ to describe $D$ as well. In particular, we use $\sigma$ as a coordinate of $\partial D=S^{1}$ that is identified with the $S^{1}$ entering the definition of the world-sheet $\Sigma=S^{1} \times \mathbb{R}$ of the lambda model action in (2.3).

Using the decomposition

$$
\mathcal{A}=d \tau A_{\tau}+A, \quad \hat{d}=d \tau \partial_{\tau}+d,
$$

we end up with the following action functional

$$
S=\frac{\bar{k}}{4 \pi} \int_{D \times \mathbb{R}} d \tau\left\langle-A \partial_{\tau} A+2 A_{\tau} F\right\rangle-\frac{\bar{k}}{4 \pi} \int_{\partial D \times \mathbb{R}} d \tau\left\langle A_{\tau} A\right\rangle,
$$

where $F=d A+A^{2}$ is the curvature of the 2-dimensional gauge field $A=A_{i} d x^{i}$ not to be confused with the world-sheet gauge field entering the definition of the action (2.3). Notice that we have omitted the wedge product symbol $\wedge$ in order to simplify the notation but we can put it back if required. It is also useful to work in terms of differential forms rather than in terms of components.

The Lagrangian is then given by

$$
L=\frac{\bar{k}}{4 \pi} \int_{D}\left\langle-A \partial_{\tau} A+2 A_{\tau} F\right\rangle-\frac{\bar{k}}{4 \pi} \int_{\partial D}\left\langle A_{\tau} A\right\rangle,
$$

whose arbitrary variation is as follows

$$
\delta L=\frac{\bar{k}}{2 \pi} \int_{D}\left\langle\delta A_{\tau} F+\delta A\left(D A_{\tau}-\partial_{\tau} A\right)\right\rangle+\frac{\bar{k}}{4 \pi} \int_{\partial D} d \sigma\left\langle\delta A_{\sigma} A_{\tau}-\delta A_{\tau} A_{\sigma}\right\rangle,
$$

where $D(*)=d(*)+[A, *]$ is a covariant derivative. From this we find the bulk equations of motion

$$
F=0, \quad \partial_{\tau} A-D A_{\tau}=0, \quad \text { on } \quad D
$$

stating that the 3 -dimensional gauge field $\mathcal{A}$ is flat, as well as the boundary equations of motion

$$
\left\langle\delta A_{\sigma} A_{\tau}-\delta A_{\tau} A_{\sigma}\right\rangle=0 \quad \text { on } \quad \partial D
$$


which must be solved consistently in order to obtain the field configurations minimizing the action. A possible useful solution to the boundary equations of motion is to demand that $A_{\tau}=\xi A_{\sigma}$, for some constant factor $\xi$ or the more general boundary conditions considered in [68]. However, as we shall see, for the lambda models they are automatically satisfied.

The Lagrangian (3.6) is already written in Hamiltonian form. The Hamiltonian includes a boundary term and it is given by

$$
H=-\frac{\bar{k}}{2 \pi} \int_{D}\left\langle A_{\tau} F\right\rangle+\frac{\bar{k}}{4 \pi} \int_{\partial D}\left\langle A_{\tau} A\right\rangle .
$$

The fundamental Poisson brackets extracted from (3.6) are found to be ${ }^{11}$

$$
\left\{\begin{array}{l}
\stackrel{1}{A}_{i}(x), \stackrel{2}{A} \\
j
\end{array}(y)\right\}=\frac{2 \pi}{\bar{k}} \epsilon_{i j} C_{12} \delta_{x y}, \quad\left\{\stackrel{1}{A}_{\tau}(x), \stackrel{2}{P}_{\tau}(y)\right\}=C_{12} \delta_{x y}
$$

and for arbitrary functions of $A_{i}$, they generalize to ${ }^{12}$

$$
\{F(A), G(A)\}=\frac{2 \pi}{\bar{k}} \epsilon_{i j} \int_{D} d^{2} x \frac{\delta F(A)}{\delta A_{i}^{A}(x)} \eta^{A B} \frac{\delta G(A)}{\delta A_{j}^{B}(x)} .
$$

The definition of the functional derivatives $\delta F / \delta A$ to be used in the bracket above is subtle because of the presence of boundaries $[56,58]$. To find them, we start with the variation $\delta F(A)$ and subsequently find a way to write the result as an integral over the disc $D$ only. For example, for the Hamiltonian we find that

$$
\delta H=-\frac{\bar{k}}{2 \pi} \int_{D}\left\langle\delta A_{\tau} F+\delta A D A_{\tau}\right\rangle-\frac{\bar{k}}{4 \pi} \int_{\partial D} d \sigma\left\langle\delta A_{\sigma} A_{\tau}-\delta A_{\tau} A_{\sigma}\right\rangle .
$$

Then, to cancel the boundary term we must use the boundary equations of motion (3.9) in order to obtain the desired well-behaved result

$$
\delta H=-\frac{\bar{k}}{2 \pi} \int_{D}\left\langle\delta A_{\tau} F+\delta A D A_{\tau}\right\rangle .
$$

Now we are ready to consider the Dirac procedure. There is a primary constraint

$$
P_{\tau} \approx 0
$$

whose stability condition leads to a secondary constraint

$$
F \approx 0
$$

which is nothing but the first bulk equation of motion in (3.8). To study the secondary constraints we better introduce the general quantity

$$
G_{0}(\eta)=\frac{\bar{k}}{2 \pi} \int_{D}\langle\eta F\rangle
$$

\footnotetext{
${ }^{11}$ The $2+1$ notation used in this paper is: $\epsilon_{12}=1$ and $\delta_{x y}=\delta^{(2)}(x-y)$. For the Lie (super)-algebra we define $\eta_{A B}=\left\langle T_{A}, T_{B}\right\rangle, C_{12}=\eta^{A B} T_{A} \otimes T_{B}$ and $\stackrel{1}{u}=u \otimes I, \stackrel{2}{u}=I \otimes u$, etc.

${ }^{12}$ For arbitrary functions of $A_{\tau}$, the Poisson bracket is obvious and will not be written.
} 
and compute its variation assuming that the test functions $\eta$ are independent of the phase space variables $\left\{A_{\tau}, A_{i}\right\}$. We find that

$$
\delta G_{0}(\eta)=\frac{\bar{k}}{2 \pi} \int_{D}\langle\delta A D \eta\rangle+\frac{\bar{k}}{2 \pi} \delta \int_{\partial D}\langle\eta A\rangle
$$

Then, the constraint with a well-defined functional derivative is actually the shifted one

$$
G(\eta)=G_{0}(\eta)+G_{1}(\eta), \quad G_{1}(\eta)=-\frac{\bar{k}}{2 \pi} \int_{\partial D}\langle\eta A\rangle .
$$

Using this we can show that the action of the shifted constraint is a gauge transformation

$$
\delta_{\eta} A \equiv\{A, G(\eta)\}=-D \eta
$$

and that the second equation of motion in (3.8) can be written as a special gauge transformation

$$
\partial_{\tau} A=\delta_{\left(-A_{\tau}\right)} A,
$$

because $\delta H=\delta G\left(-A_{\tau}\right)$. Then, despite of the fact that $A_{\tau}$ is a phase space coordinate both quantities turn out to generate the same action.

The constraint algebra is now given by the bracket

$$
\begin{aligned}
\{G(\eta), G(\bar{\eta})\} & =\frac{\bar{k}}{2 \pi} \int_{D}\langle D \eta D \bar{\eta}\rangle \\
& =G([\eta, \bar{\eta}])+\frac{\bar{k}}{2 \pi} \int_{\partial D}\langle\eta d \bar{\eta}\rangle
\end{aligned}
$$

after some standard manipulations, showing that when the test functions or their derivatives do not vanish on $\partial D$, the shifted constraints are actually second class because of the presence of the boundary. On the other hand, the former constraints $G_{0}(\eta)$ are also second class [56] for the same kind of test functions and this means that no extra gauge-fixing conditions are required allowing to introduce a Dirac bracket for the constraints $F \approx 0$ in a natural way. In this paper we will restrict to this kind of improper $[58,59]$ test/gauge functions only.

Now we can show that

$$
\begin{aligned}
\{G(\eta), H\} & =-G\left(\left[\eta, A_{\tau}\right]\right)-\frac{\bar{k}}{2 \pi} \int_{\partial D}\left\langle\eta d A_{\tau}\right\rangle \\
& \approx-\frac{\bar{k}}{2 \pi} \int_{\partial D}\left\langle\eta D A_{\tau}\right\rangle .
\end{aligned}
$$

Using this result we can find the time evolution of the secondary constraints. We obtain

$$
\begin{aligned}
\frac{d G(\eta)}{d \tau} & \approx\{G(\eta), H\}+\int_{\partial D} \frac{\delta G(\eta)}{\delta \eta^{A}} \partial_{\tau} \eta^{A} \\
& \approx \frac{\bar{k}}{2 \pi} \int_{\partial D} d \sigma\left\langle\eta F_{\tau \sigma}\right\rangle-\frac{\bar{k}}{2 \pi} \int_{\partial D} d \sigma \partial_{\tau}\left\langle\eta A_{\sigma}\right\rangle
\end{aligned}
$$


and after pulling $\partial_{\tau}$ outside the integral as $\frac{d}{d \tau}$, we get the final result

$$
\frac{d G_{0}(\eta)}{d \tau} \approx \frac{\bar{k}}{2 \pi} \int_{\partial D} d \sigma\left\langle\eta F_{\tau \sigma}\right\rangle
$$

which vanish if we demand that

$$
F_{\tau \sigma} \approx 0 \text { on } \partial D \text {. }
$$

This is the second bulk equation of motion in (3.8) (or constraint) with $i=\sigma$ extended to $\partial D$. We will come back to this important boundary constraint later on. There are no tertiary constraints.

Following [56], we now write down the non-zero Poisson algebra for the quantities $G_{0}, G_{1}, G$ on the constraint surface $F \approx 0$. It is given by ${ }^{13}$

$$
\begin{aligned}
\left\{G_{0}(\eta), G_{0}(\bar{\eta})\right\} & \approx-\frac{\bar{k}}{2 \pi} \int_{\partial D} d \sigma\left\langle\eta, D_{\sigma} \bar{\eta}\right\rangle, \\
\left\{G_{0}(\eta), G_{1}(\bar{\eta})\right\} & \approx \frac{\bar{k}}{2 \pi} \int_{\partial D} d \sigma\left\langle\eta, D_{\sigma} \bar{\eta}\right\rangle, \\
\{G(\eta), G(\bar{\eta})\} & \approx \frac{\bar{k}}{2 \pi} \int_{\partial D} d \sigma\left\langle\eta, D_{\sigma} \bar{\eta}\right\rangle .
\end{aligned}
$$

In order to impose $F \approx 0$ strongly, we used Dirac brackets. The only non-zero Dirac brackets are easily found to be

$$
\begin{aligned}
\left\{G_{1}(\eta), G_{1}(\bar{\eta})\right\}^{*} & =\frac{\bar{k}}{2 \pi} \int_{\partial D} d \sigma\left\langle\eta, D_{\sigma} \bar{\eta}\right\rangle, \\
\{G(\eta), G(\bar{\eta})\}^{*} & =\frac{\bar{k}}{2 \pi} \int_{\partial D} d \sigma\left\langle\eta, D_{\sigma} \bar{\eta}\right\rangle,
\end{aligned}
$$

which are consistent with setting $F=0$ strongly. From this follows that only the boundary contribution $G_{1}(\eta)$ remains. Then, on the constrained surface, (3.20) takes the form [56]

$$
\delta_{\eta} A \equiv\left\{A, G_{1}(\eta)\right\}^{*}=-D \eta,
$$

which in turn imply that the second set of equations of motion in (3.8) can be written as

$$
\partial_{\tau} A=\delta_{\left(-A_{\tau}\right)} A=\left\{A, H^{*}\right\}^{*},
$$

because $\delta H^{*}=\delta G_{1}\left(-A_{\tau}\right)$. In showing this last result we have used the boundary equations of motion (3.9) as required before and the restriction of (3.10) to the constrained surface given by

$$
H^{*}=\frac{\bar{k}}{4 \pi} \int_{\partial D} d \sigma\left\langle A_{\tau} A_{\sigma}\right\rangle .
$$

Then, the time evolution on the constraint surface takes the correct form under the Dirac bracket but now in terms of the boundary Hamiltonian.

\footnotetext{
${ }^{13}$ It is important to mention at this point that $G_{0}$ and $G_{1}$ separately also have well-defined functional variations, as shown in [56] after a careful treatment of boundary terms.
} 
To identify the reduced phase space coordinates we replace (3.11) by its Dirac bracket, ${ }^{14}$ but this is equivalent to pulling the symplectic form associated to (the first bracket in) (3.11) back to the constraint surface $[45,46]$. Namely,

$$
\omega^{*}=\left.\frac{\bar{k}}{4 \pi} \int_{D}\langle\delta A \wedge \delta A\rangle\right|_{A=-d \Psi \Psi^{-1}}=-\frac{\bar{k}}{4 \pi} \int_{\partial D} d \sigma\left\langle\delta A_{\sigma} \wedge D_{\sigma}^{-1} \delta A_{\sigma}\right\rangle
$$

The Poisson bracket that follows ${ }^{15}$ from this reduced symplectic form is equivalent to (3.28) and after eliminating the test functions, we reveal the Kac-Moody algebra structure

$$
\left\{\stackrel{1}{A}_{\sigma}(\sigma), \stackrel{2}{A_{\sigma}}\left(\sigma^{\prime}\right)\right\}^{*}=\frac{2 \pi}{\bar{k}}\left(\left[C_{12}, \stackrel{2}{A}_{\sigma}\left(\sigma^{\prime}\right)\right] \delta_{\sigma \sigma^{\prime}}+C_{12} \delta_{\sigma \sigma^{\prime}}^{\prime}\right)
$$

of the associated WZW model on $\partial D$. In this sense we say that the phase space information of the CS theory is now completely stored on its boundary theory. Indeed, the reduced phase space is described by the data $\left(\left.A_{\sigma}\right|_{\partial D}, H^{*},\{\cdot, \cdot\}^{*}\right)$. The time evolution of $A_{\sigma}$ can be put in Hamiltonian form and it is given precisely by the boundary constraints (3.26), which as we shall see below are equivalent to the string lambda model equations of motion. What we have presented here is nothing but the Hamiltonian version of the (well-known) relation that exists between the CS and the WZW theories that is found in the literature but in a different guise.

We are now ready to introduce the dependence of the spectral parameter $z$. Not surprisingly, the $( \pm)$ sub-index introduced above make reference to the two special points $z_{ \pm}=\lambda^{ \pm 1 / 2}$ in the complex plane parameterized by $z$ and introduced in the last section. We now make use of the twisted loop superalgebra structure (2.2) and consider the problem of finding a $z$-dependent 2-dimensional gauge field $A(z)$ on the disc $D$ satisfying the following two conditions. ${ }^{16}$

$$
A\left(z_{ \pm}\right)=A_{( \pm)} \quad \text { and } \quad \Phi(A(z))=A(i z)
$$

The answer we will consider here (recall that $\left.A(z)=A_{i}(z) d x^{i}, i=1,2\right)$ is given by

$$
A(z)=f_{-}(z) \bar{\Omega}\left(z / z_{+}\right) A_{(+)}-f_{+}(z) \bar{\Omega}\left(z / z_{-}\right) A_{(-)}, \quad f_{ \pm}(z)=\frac{\left(z^{4}-z_{ \pm}^{4}\right)}{\left(z_{+}^{4}-z_{-}^{4}\right)}
$$

where

$$
\bar{\Omega}(z)=P^{(0)}+z^{-3} P^{(1)}+z^{-2} P^{(2)}+z^{-1} P^{(3)}
$$

is another projector not to be confused with the one defining the GS lambda model (2.4) above. Actually, this same projector appears for both the GS [25] and the hybrid superstring [8] and leads to the same Maillet bracket when (2.16) or (3.33) is used. Then, both superstring formulations are equivalent at this level of analysis.

\footnotetext{
${ }^{14}$ We will not consider phase space functionals depending on $P_{\tau}$.

${ }^{15}$ The symplectic form operator is formally identified as $\hat{\omega}=D_{\sigma}^{-1}$ with an associated Poisson operator given by $\hat{\theta}=D_{\sigma}$ that is responsible for the Kac-Moody algebra structure.

${ }^{16}$ Here we are considering only the horizontal fields $A_{( \pm)}$, but it works exactly the same way for $\mathcal{A}_{( \pm)}$so we can consider $\mathcal{A}(z)$ instead from the beginning and then restrict it to the disc.
} 
Using this $z$-dependent gauge field we can gather both Poisson brackets on the l.h.s. of (3.11) into a single interpolating one

$$
\left\{{ }^{1} A_{i}(x, z), \stackrel{2}{A}_{j}(y, w)\right\}=-2 \mathfrak{s}_{12}(z, w) \epsilon_{i j} \delta_{x y}
$$

which, as we shall see, is the precursor of the Maillet bracket. From here we can appreciate that it is the Chern-Simons Poisson structure $\left(\left\{A_{i}, A_{j}\right\} \approx \epsilon_{i j}\right.$ and not $\left.\left\{A_{i}, A_{j}\right\} \approx \delta_{i j}\right)$ the one responsible for the non skew-symmetry of the $R$-matrix entering the Maillet bracket and the very source of its non ultralocality. In this calculation we face exactly the same situation of $[8]$ and find that

$$
\mathfrak{s}_{12}(z, w)=-\frac{1}{z^{4}-w^{4}} \sum_{j=0}^{3}\left\{z^{j} w^{4-j} C_{12}^{(j, 4-j)} \varphi_{\lambda}^{-1}(w)-z^{4-j} w^{j} C_{12}^{(4-j, j)} \varphi_{\lambda}^{-1}(z)\right\},
$$

where

$$
\varphi_{\lambda}(z)=\frac{k}{\pi} \frac{\left(\lambda^{-2}-\lambda^{2}\right)}{\left(z^{-2}-z^{2}\right)^{2}-\left(\lambda^{-1}-\lambda\right)^{2}}
$$

is the lambda deformed twisting function. Notice that the two special points $z_{ \pm}=\lambda^{ \pm 1 / 2}$ are poles of $\varphi_{\lambda}(z)$. In retrospective, we realize that our theory (3.2) actually consist of two Chern-Simons theories with opposite levels attached to the poles $z_{ \pm}$of (3.39) in the complex plane or the Riemann sphere after compactification.

The symmetric operator $\mathfrak{s}_{12}(z, w)$ satisfy

$$
\mathfrak{s}_{12}\left(z_{ \pm}, z_{ \pm}\right)=\mp \frac{\pi}{k} C_{12}=-\frac{\pi}{\bar{k}} C_{12}, \quad \mathfrak{s}_{12}\left(z_{ \pm}, z_{\mp}\right)=0
$$

as required for the Poisson algebra (3.37) to reduce to (3.11) at the poles. It also satisfy

$$
\lim _{\lambda \rightarrow 0} \mathfrak{s}_{12}(z, w)=-\frac{\pi}{k} C_{12}^{(00)}
$$

but we still do not have a proper interpretation for this limit which corresponds to the ultra-localization limit of the lambda models and that is deeply related to the Pohlmeyer reduction of the $A d S_{5} \times S^{5}$ GS superstring [42]. As customary, we will refer to the limits $\lambda \rightarrow 0$ and $\lambda \rightarrow 1$ as the sine-Gordon and sigma model limits, respectively.

For arbitrary functions and their differentials

$$
F(A)=(F, A)_{\varphi_{\lambda}}, \quad \lim _{t \rightarrow 0} \frac{d}{d t} F(A+t X)=(d F, X)_{\varphi_{\lambda}},
$$

the Poisson bracket (3.37) generalize to

$$
\{F(A), G(A)\}=(R(d F), d G)_{\varphi_{\lambda}}+(d F, R(d G))_{\varphi_{\lambda}},
$$

where $R= \pm\left(\Pi_{\geq 0}-\Pi_{<0}\right)$ is the usual AKS $R$-operator defined in terms of the projectors $\Pi$ that act on elements of $\hat{\mathfrak{f}}$. The definitions are as follows: for a $z$-dependent 2 -form on $D$ constructed from the pair $X$ and $Y$, we have

$$
(X, Y)_{\varphi_{\lambda}}=\int_{D}\langle X(x), Y(x)\rangle_{\varphi_{\lambda}}
$$


where

$$
\langle X(x), Y(x)\rangle_{\varphi_{\lambda}}=\oint_{0} \frac{d z}{2 \pi i z} \varphi_{\lambda}(z)\langle X(x, z), Y(x, z)\rangle
$$

is the twisted inner product on the loop superalgebra $\widehat{\mathfrak{f}}$ for fixed $x$. For example, the functions

$$
F_{( \pm)}(A)=\int_{D}\left\langle A_{( \pm)} \wedge \eta_{( \pm)}\right\rangle=\int_{D} d^{2} x\left\langle A_{i( \pm)} \eta_{j( \pm)}\right\rangle \epsilon_{i j},
$$

where $\eta_{( \pm)}$are two test 1 -forms can be written in terms of (3.44) as

$$
F_{( \pm)}(A)=\left(\eta_{( \pm)}, A\right)_{\varphi_{\lambda}},
$$

with

$$
\eta_{( \pm)}(z)=\varphi_{\lambda}^{-1}(z) z_{ \pm}^{4}\left(z^{-4}+z_{\mp}^{4}\right)\left\{\eta_{( \pm)}^{(0)}+z z_{\mp} \eta_{( \pm)}^{(1)}+z^{2} z_{\mp}^{2} \eta_{( \pm)}^{(2)}+z^{3} z_{\mp}^{3} \eta_{( \pm)}^{(3)}\right\} .
$$

Now, because of the functions $F_{( \pm)}(A)$ are linear in $A_{( \pm)}$, their differentials are $d F_{( \pm)}(z)=$ $\eta_{( \pm)}(z)$. Using these expressions in (3.43), we recover ${ }^{17}$ the first expression in (3.11).

Now we can compute the $z$-dependent boundary algebra after imposing the constraints (3.16) strongly. We take $i=\sigma$ in (3.35) and use (3.33). Explicitly,

$$
\begin{aligned}
A_{\sigma}(z)=\frac{\left(z^{4}-z_{-}^{4}\right)}{\left(z_{+}^{4}-z_{-}^{4}\right)} & \left\{A_{\sigma(+)}^{(0)}+\frac{z_{+}^{3}}{z^{3}} A_{\sigma(+)}^{(1)}+\frac{z_{+}^{2}}{z^{2}} A_{\sigma(+)}^{(2)}+\frac{z_{+}}{z} A_{\sigma(+)}^{(3)}\right\} \\
& -\frac{\left(z^{4}-z_{+}^{4}\right)}{\left(z_{+}^{4}-z_{-}^{4}\right)}\left\{A_{\sigma(-)}^{(0)}+\frac{z_{-}^{3}}{z^{3}} A_{\sigma(-)}^{(1)}+\frac{z_{-}^{2}}{z^{2}} A_{\sigma(-)}^{(2)}+\frac{z_{-}}{z} A_{\sigma(-)}^{(3)}\right\} .
\end{aligned}
$$

As a consequence of the Kac-Moody algebra structure (3.33) and once more following [8], we obtain the Maillet bracket

$$
\begin{aligned}
\left\{A_{\sigma}^{1}(\sigma, z), A_{\sigma}\left(\sigma^{\prime}, w\right)\right\}^{*}= & -\left[\mathfrak{r}_{12}, A_{\sigma}(\sigma, z)+\stackrel{2}{A_{\sigma}}\left(\sigma^{\prime}, w\right)\right] \delta_{\sigma \sigma^{\prime}} \\
& +\left[\mathfrak{s}_{12}, A_{\sigma}(\sigma, z)-\stackrel{A}{A}_{\sigma}\left(\sigma^{\prime}, w\right)\right] \delta_{\sigma \sigma^{\prime}}-2 \mathfrak{s}_{12} \delta_{\sigma \sigma^{\prime}}^{\prime}
\end{aligned}
$$

where

$$
\mathfrak{r}_{12}(z, w)=\frac{1}{z^{4}-w^{4}} \sum_{j=0}^{3}\left\{z^{j} w^{4-j} C_{12}^{(j, 4-j)} \varphi_{\lambda}^{-1}(w)+z^{4-j} w^{j} C_{12}^{(4-j, j)} \varphi_{\lambda}^{-1}(z)\right\},
$$

is the anti-symmetric part of the $R$-matrix. This is the same algebra obtained with the extended Lax operator (2.16) and we now identify $A_{\sigma}(z)=\mathscr{L}^{\prime}(z)$. The bracket satisfy the Jacobi identity and reduce to (3.33) at the poles. The sine-Gordon limit (3.41) applied to (3.50) is a continuous version of the alleviating mechanism introduced in [18] so the non ultralocality of the Maillet algebra is still present for (semi)-symmetric cosets. Then, in order to suppress the $\delta_{\sigma \sigma^{\prime}}^{\prime}$ completely for any value of $\lambda$, we must go to a higher dimension.

\footnotetext{
${ }^{17}$ The $R$-operator with the minus sign is the one that reproduce the first Poisson bracket expression in (3.11) explicitly.
} 
Alternatively, by setting

$$
\mathfrak{r}_{12}=\frac{1}{2}\left(R_{12}-R_{12}^{*}\right), \quad \mathfrak{s}_{12}=-\frac{1}{2}\left(R_{12}+R_{12}^{*}\right),
$$

we can write ${ }^{18}$

$$
\left\{\stackrel{1}{A_{\sigma}}(\sigma, z), \stackrel{2}{A_{\sigma}}\left(\sigma^{\prime}, w\right)\right\}^{*}=-\left[R_{12}, \stackrel{1}{A_{\sigma}}(\sigma, z)\right] \delta_{\sigma \sigma^{\prime}}+\left[R_{12}^{*}, \stackrel{2}{A_{\sigma}}\left(\sigma^{\prime}, w\right)\right] \delta_{\sigma \sigma^{\prime}}+\left(R_{12}+R_{12}^{*}\right) \delta_{\sigma \sigma^{\prime}}^{\prime},
$$

where

$$
R_{12}(z, w)=\frac{2}{z^{4}-w^{4}} \sum_{j=0}^{3} z^{j} w^{4-j} C_{12}^{(j, 4-j)} \varphi_{\lambda}^{-1}(w), \quad R_{12}^{*}(z, w)=R_{21}(w, z) .
$$

For arbitrary functions of $A_{\sigma},(3.50)$ generalize to

$$
\{F, G\}^{*}\left(A_{\sigma}\right)=-\left(A_{\sigma},[d F, d G]_{R}\right)_{\varphi_{\lambda}}+\omega(R(d F), d G)_{\varphi_{\lambda}}+\omega(d F, R(d G))_{\varphi_{\lambda}},
$$

where $[*, *]_{R}$ is the $R$-bracket on $\widehat{\mathfrak{f}}$ and

$$
\omega(X, Y)_{\varphi_{\lambda}}=\int_{\partial D} d \sigma\left\langle X(\sigma), \partial_{\sigma} Y(\sigma)\right\rangle_{\varphi_{\lambda}}
$$

is the co-cycle. The only difference when compared to (3.44) is that the inner product integration is now performed on $\partial D$, the $d \sigma$ is written explicitly and the $X, Y$ are ordinary functions on $\partial D$. Namely,

$$
(X, Y)_{\varphi_{\lambda}}=\int_{\partial D} d \sigma\langle X(\sigma), Y(\sigma)\rangle_{\varphi_{\lambda}}
$$

The bracket (3.50) can, alternatively, be written in the form

$$
\{F, G\}^{*}\left(A_{\sigma}\right)=\left(R(d F), \hat{D}_{\sigma} d G\right)_{\varphi_{\lambda}}+\left(d F, \hat{D}_{\sigma} R(d G)\right)_{\varphi_{\lambda}},
$$

where $\hat{D}_{\sigma}(*)=\partial_{\sigma}(*)+\left[A_{\sigma}(z), *\right]$. From this we identify $\hat{\theta}=\hat{D}_{\sigma} \circ R+R^{*} \circ \hat{D}_{\sigma}$, which is the analogue of the $\hat{\theta}$ in (3.33) (see footnote 15). Following the same steps we realize that (3.55) is the $z$-dependent extension of the Dirac bracket associated to (3.43) after imposing the constraints $F\left(z_{ \pm}\right)=0$. A comment is in order. Notice that we are referring to (3.55) as an extension of the Dirac bracket because $F(z)$ (the curvature of $A(z)$ ) reproduce the correct Hamiltonian constraints only when it reach the poles $z_{ \pm}$. In order to find a proper $z$-dependent constraint (if any), we probably would need to lift the action (3.1) to the loop superalgebra $\hat{\mathfrak{f}}$ and run the Dirac procedure again but as we have seen, the introduction of the spectral parameter is rather innocuous and does not introduce new degrees of freedom or fields so no new constraints are expected beyond those attached to the points $z_{ \pm}$. However, by an abuse of notation we will keep the $*$ on (3.55).

Now we are in the position to interpret the boundary equations of motion (3.9). First we note that the link between the Kac-Moody algebras (3.33) and (2.14) is through the relation

$$
A_{\sigma( \pm)}=\mp \frac{2 \pi}{k} \mathscr{J}_{\mp}=\mathscr{L}_{\sigma}\left(z_{ \pm}\right)
$$

\footnotetext{
${ }^{18} \mathrm{Up}$ to a global minus sign this is the same Maillet braket constructed in [41].
} 
where we have used (2.13) in the last equality. Now, the obvious solution to the boundary equations of motion is to identify

$$
A_{\tau( \pm)}=\mathscr{L}_{\tau}\left(z_{ \pm}\right)
$$

To see why, we rewrite (3.9) in the form

$$
\epsilon^{\mu \nu}\left\langle\delta \mathscr{L}_{\mu}\left(z_{+}\right) \mathscr{L}_{\nu}\left(z_{+}\right)-\delta \mathscr{L}_{\mu}\left(z_{-}\right) \mathscr{L}_{\nu}\left(z_{-}\right)\right\rangle=0
$$

and use the fact that the product $\left\langle\delta \mathscr{L}_{ \pm}(z) \mathscr{L}_{\mp}(z)\right\rangle$ is independent of the spectral parameter either for the Green-Schwarz or the hybrid superstring Lax Pairs (2.8) and (2.22), respectively. The PCM Lax pair also satisfy (3.61) trivially. Thus, for the lambda models the CS boundary equation of motion (3.61) is just an identity. The explicit transformation between the components of the CS gauge field on $\partial D \times \mathbb{R}$ and the lambda model gauge field on $\Sigma$ is

$$
A_{\sigma( \pm)}=\left\{\begin{array}{c}
A_{+}-\Omega A_{-} \\
\Omega^{T} A_{+}-A_{-}
\end{array}, \quad A_{\tau( \pm)}=\left\{\begin{array}{c}
A_{+}+\Omega A_{-} \\
\Omega^{T} A_{+}+A_{-}
\end{array}\right.\right.
$$

The relations (3.59) and (3.60) allow to extract an important piece of information from the boundary constraints (3.26) if we write them in the form

$$
\left[\partial_{+}+\mathscr{L}_{+}\left(z_{ \pm}\right), \partial_{-}+\mathscr{L}_{-}\left(z_{ \pm}\right)\right]=0
$$

The first conclusion is that they are precisely the lambda model Euler-Lagrange equations of motion (2.7) of the action (2.3), which also follows from a Lax pair zero curvature condition. The second conclusion is that they imply the conservation of the Poisson-Lie charges $m\left(z_{ \pm}\right)$in (1.1) (see [24]). The boundary degrees of freedom of the double CS theory on $\mathfrak{p s u}(2,2 \mid 4)$ are described by the $A d S_{5} \times S^{5}$ lambda model action (2.3). This conclusion also apply to the other models we have considered so far.

It is important to realize that the identification between (3.50) and (2.17) holds for the extended Lax operator. At this point the result is rather generic (recall we only required (3.34) in the construction) and in order to consider a particular lambda model the omega projector $\Omega$ must be specified as it determines the Hamiltonian constraint structure of the theory under the Dirac procedure. In other words, it determines the decomposition

$$
\mathscr{L}^{\prime}(z)=\mathscr{L}(z)+\text { constraints }
$$

from where the current algebra of the deformed dual currents $I_{ \pm}$can be computed. It matches the one found by using the direct relation $(2.15)$. See $[60,61]$ for the the conventional GS superstring and [8] for the lambda model of the hybrid superstring. It is remarkable that the CS theory reproduce the Hamiltonian Lax connection as it has interesting properties, see [61] for the specific case GS formulation in relation to the involution properties of the charges extracted from the monodromy matrix. Notice that the twisted loop and the Kac-moody superalgebras combined are the ones responsible for introducing the $R$-matrix with spectral parameter. 
There is a certain amount of freedom in the construction of the current (3.35). For instance, one could consider several copies of the Chern-Simons actions (3.2) in the definition of the theory involving different WZW levels $k^{\prime} s$, which would alter the first condition in (3.34) or we can also consider a consistent algebraic modification to the second condition in (3.34). Either case, this could lead to more general twisting functions and $\mathfrak{r} / \mathfrak{s}$ tensors and to novel multiparametric lambda deformations of string sigma models. An example of a consistent modification of the second condition, when $\mathfrak{f}$ is a bosonic Lie algebra, is the PCM. For this case, the loop superalgebra has $\Phi=I$, i.e. no $\mathbb{Z}_{2}$ grading and

$$
\mathscr{L}^{\prime}(z)=\mathscr{L}(z)=f_{-}(z) \mathscr{J}_{+}+f_{+}(z) \mathscr{J}_{-},
$$

i.e. no first class constraints. The explicit form of the functions $f_{ \pm}(z)$ for this case can be found by working out explicitly the Lax pair representation. The $\mathfrak{r} / \mathfrak{s}$ tensors as well as the twisting function $\varphi_{\lambda}(z)$ and their poles $z_{ \pm}$become those of the lambda deformation of the PCM [24].

On the constrained surface, the complete Hamiltonian (3.31) is given by (we drop the $*$ )

$$
H=\frac{k}{4 \pi} \int_{\partial D} d \sigma\left\langle A_{\tau}\left(z_{+}\right) A_{\sigma}\left(z_{+}\right)-A_{\tau}\left(z_{-}\right) A_{\sigma}\left(z_{-}\right)\right\rangle,
$$

which should be compared with the lambda model Hamiltonian in (2.21). Mimicking (2.21), we define the momentum generator

$$
P=\frac{k}{8 \pi} \int_{\partial D} d \sigma\left\langle\left(A_{\tau}^{2}\left(z_{+}\right)+A_{\sigma}^{2}\left(z_{+}\right)\right)-\left(A_{\tau}^{2}\left(z_{-}\right)+A_{\sigma}^{2}\left(z_{-}\right)\right)\right\rangle
$$

which commutes with $H$ under the bracket (3.33). The opposite signs of the levels at $( \pm)$ are important in showing this. We can recover the Virasoro algebra structure of the lambda models if we define the usual $T_{ \pm \pm}$components as in (2.20).

Finally, the boundary equations of motion (3.9) can be understood as a condition dictating the form of the Lax pair and the boundary constraints (3.26) as a condition dictating the dynamics of the system because of their equivalence to the lambda model Euler-Lagrange equations of motion. This is precisely the content of equation (2.11) which summarizes the integrability properties of the system. The flatness condition as well as the analytic properties of $\mathscr{L}_{ \pm}(z)$ are known to be preserved by the action of the group of dressing transformations $[25,62,63]$ that can be seen now as an infinite dimensional symmetry group of the boundary theory.

\section{Concluding remarks}

The main goal of this paper was to show how the lambda model (2.3) can be reformulated as a double Chern-Simons theory (3.1) and how the Lax pair representation and the Maillet bracket structure of the lambda model phase space emerge from the CS theory point of view. The strategy is to trade the non-ultralocal Maillet bracket (3.50) by the ultralocal CS bracket (3.37) at the expense of introducing a couple of new constraints (3.16), so the price to pay for the elimination of the problematic $\delta_{\sigma \sigma^{\prime}}^{\prime}$ term is to deal with the quadratic second 
class constraints $F\left(z_{ \pm}\right) \approx 0$ on the disc. To obtain a string theory the Virasoro constraints $T_{ \pm \pm} \approx 0$ as well as the gauge fixing of the kappa symmetry must be taken into account and, fortunately, both can be handled at the same time by means of the light-cone dressing gauge introduced in [25], which reduce to picking a particular orbit of the $\lambda$-deformed BMN vacuum solution under the action of the dressing group $\Psi(z)=\chi(z) \Psi_{0}(z)$.

One may wonder what is to be gained in complicating even more the phase space structure of the string lambda models by introducing the constraints $F\left(z_{ \pm}\right) \approx 0$ proper of the CS setting. On the one hand, by doing this we can, not only to suppress completely the non ultralocality of all known $\lambda$-models (i.e. $\mathrm{PCM}_{\lambda}, \mathrm{F} / \mathrm{G}_{\lambda}$, GS on $A d S_{5} \times_{\lambda} S^{5}$ and hybrid on $A d S_{2} \times_{\lambda} S^{2}$ ) but also to do it for any (generic) value of the deformation parameter $\lambda$. On the other hand, the new theory being of the CS type can, in principle, be quantized in a number of ways. In particular, by employing a (disc) lattice algebra regularization [30-34] or by a path integral approach [44, 47-49]. However, for superstrings we have the added complication that the CS theories are defined on Lie superalgebras, which is not a common feature of conventional CS theories. ${ }^{19}$ The problem of quantizing our Hamiltonian double CS theory in the presence of the spectral parameter, i.e. the quantization of the Poisson bracket (3.37), is currently under investigation [35] based on the combinatorial quantization approach of [30-34].

Several natural questions raise from these first steps and in what follows we mention some of them:

One potential application of this approach would be to study finite size effects. For $r \rightarrow \infty$, the boundary decompactifies and $\Sigma=S^{1} \times \mathbb{R} \rightarrow \mathbb{R}^{1,1}$. In this situation, the lambda model action must be carefully modified along the lines of $[54,55]$ in order to accommodate the new boundary conditions. In this limit though, asymptotic states and their S-matrix can be defined but for finite $r$ (to our knowledge) not much is known. It would be enlightening to study what the CS theory could tell us about the quantum integrability of the $1+1$ theory for any value of $r$. A strategy for quantization would be to quantize the ultralocal theory on the disc and afterwards project the quantum theory to the boundary in some sort of holographic way (by imposing all the constraints). This is opposite to the usual symplectic reduction approach of first enforcing the constraints $F=0$ classically and then quantizing the remaining degrees of freedom.

The fundamental objects of our double CS theory would be the Wilson loops with spectral parameter

$$
W_{R}(C ; z)=\left\langle P \exp \left(-\oint_{C} \mathcal{A}(z)\right)\right\rangle_{R}
$$

for $C$ a knot in $M=D \times \mathbb{R}$ and $R$ a particular representation. If $C$ is a horizontal curve constrained to $\partial D$ and wrapping it once, then we obtain ${ }^{20}$

$$
W_{R}\left(S^{1} ; z\right)=\left\langle P \exp \left(-\int_{S^{1}} d \sigma \mathscr{L}^{\prime}(\sigma ; z)\right)\right\rangle_{R}
$$

\footnotetext{
${ }^{19}$ Superalgebra CS theories have been considered before in the literature albeit in a different context. See e.g. $[36,37]$.

${ }^{20}$ Other important objects would be the vertical $z$-dependent Wilson lines but at this moment their meaning is less clear.
} 
that is related to the monodromy of the extended Lax operator (2.16) and if we take $z=z_{ \pm}$, then we get

$$
W_{R}\left(S^{1} ; z_{ \pm}\right)=\left\langle P \exp \left( \pm \frac{2 \pi}{k} \int_{S^{1}} d \sigma \mathscr{J}_{\mp}(\sigma)\right)\right\rangle_{R}
$$

that is related to the monodromy of the two Kac-Moody currents $\mathscr{J}_{ \pm}$as in (1.1), leading to quantum groups $[24,26]$. We should then expect a natural affine quantum group symmetry enhancement in our theory in terms of a quantum $R(z)$-matrix that (hopefully) is related to (3.54) in the classical limit. Recently, in [64] it is shown that this enhancement do occur for the eta models and this is done by expanding the monodromy matrix around the poles of the eta-deformed twisting function $\varphi_{\eta}(z)$ so it is reasonable to expect that this must be true for the lambda models as well as both theories are, in a sense, complementary. Another issue is related to the computation of the (classical/quantum) algebra of $z$-dependent Wilson loops (4.1) defined on horizontal curves corresponding to the continuation of (4.2) into the interior of $D$. At this point it is too premature to make strong statements about its properties or even its existence but the results of [40], where a slightly similar situation is considered, suggest this algebra can be found ${ }^{21}$ precisely by exploiting the lattice simulation approach of [30-34].

From (3.37) and (3.43), we realize that the Poisson structure is related to the symmetric operator $R+R^{*}$. This suggest a possible lift of the action (3.5) to the twisted loop superalgebra $\hat{\mathfrak{f}}$ in terms of the inner product (3.44). The kinetic term in the Lagrangian (3.6) should, in principle, be replaced by something of the form

$$
L \sim \int_{D}\left\langle A \wedge\left(R+R^{*}\right)^{-1} \partial_{\tau} A\right\rangle_{\varphi_{\lambda}}
$$

but we have not succeeded in showing it. In any case, it would be interesting to study if there is a connection of our CS formulation with the CS construction of lattice models presented in [65-67]. In particular, if the action (2.8) of the paper [65] could be related to our action (3.1) for a 1-form $\omega \sim d z \varphi_{\lambda}(z) / z$.

A natural variation of our construction would be to investigate if the formulation in which the actions $S_{(+)}$and $S_{(-)}$are complex conjugated could be related to the eta models and if the results of [68] could be applied to relate both formulations.

The last question is how enters the lambda model dilaton field in the CS formulation after quantization is performed.

We will report on some of these questions elsewhere.

\section{Acknowledgments}

The author would like thank T. J. Hollowood and J. L. Miramontes for valuable discussions and collaboration.

Open Access. This article is distributed under the terms of the Creative Commons Attribution License (CC-BY 4.0), which permits any use, distribution and reproduction in any medium, provided the original author(s) and source are credited.

\footnotetext{
${ }^{21}$ See [38] an references therein as well as [39] for the standard $z$-independent case.
} 


\section{References}

[1] C. Klimčík, Yang-Baxter $\sigma$-models and dS/AdS T duality, JHEP 12 (2002) 051 [hep-th/0210095] [INSPIRE].

[2] K. Sfetsos, Integrable interpolations: From exact CFTs to non-Abelian T-duals, Nucl. Phys. B 880 (2014) 225 [arXiv:1312.4560] [INSPIRE].

[3] F. Delduc, M. Magro and B. Vicedo, On classical q-deformations of integrable $\sigma$-models, JHEP 11 (2013) 192 [arXiv:1308.3581] [INSPIRE].

[4] F. Delduc, M. Magro and B. Vicedo, An integrable deformation of the $A d S_{5} \times S^{5}$ superstring action, Phys. Rev. Lett. 112 (2014) 051601 [arXiv: 1309.5850] [INSPIRE].

[5] F. Delduc, M. Magro and B. Vicedo, Derivation of the action and symmetries of the q-deformed $A d S_{5} \times S^{5}$ superstring, JHEP 10 (2014) 132 [arXiv:1406.6286] [INSPIRE].

[6] T.J. Hollowood, J.L. Miramontes and D.M. Schmidtt, Integrable Deformations of Strings on Symmetric Spaces, JHEP 11 (2014) 009 [arXiv:1407.2840] [INSPIRE].

[7] T.J. Hollowood, J.L. Miramontes and D.M. Schmidtt, An Integrable Deformation of the $A d S_{5} \times S^{5}$ Superstring, J. Phys. A 47 (2014) 495402 [arXiv:1409.1538] [inSPIRE].

[8] D.M. Schmidtt, Exploring The Lambda Model Of The Hybrid Superstring, JHEP 10 (2016) 151 [arXiv: 1609.05330] [INSPIRE].

[9] B. Hoare, T.J. Hollowood and J.L. Miramontes, A Relativistic Relative of the Magnon S-matrix, JHEP 11 (2011) 048 [arXiv:1107.0628] [INSPIRE].

[10] B. Hoare, T.J. Hollowood and J.L. Miramontes, $q$-Deformation of the AdS $S_{5} x S^{5}$ Superstring S-matrix and its Relativistic Limit, JHEP 03 (2012) 015 [arXiv:1112.4485] [INSPIRE].

[11] B. Hoare, T.J. Hollowood and J.L. Miramontes, Bound States of the q-Deformed Ad $S_{5} x S^{5}$ Superstring S-matrix, JHEP 10 (2012) 076 [arXiv:1206.0010] [INSPIRE].

[12] L.D. Faddeev and N. Yu. Reshetikhin, Integrability of the Principal Chiral Field Model in (1+1)-dimension, Annals Phys. 167 (1986) 227 [INSPIRE].

[13] L. Freidel and J.M. Maillet, Quadratic algebras and integrable systems, Phys. Lett. B 262 (1991) 278 [INSPIRE].

[14] L. Hlavaty and A. Kundu, Quantum integrability of nonultralocal models through Baxterization of quantized braided algebra, Int. J. Mod. Phys. A 11 (1996) 2143 [hep-th/9406215] [INSPIRE].

[15] M. Semenov-Tian-Shansky and A. Sevostyanov, Classical and quantum nonultralocal systems on the lattice, hep-th/9509029 [INSPIRE].

[16] V.V. Bazhanov, S.L. Lukyanov and A.B. Zamolodchikov, Integrable structure of conformal field theory, quantum KdV theory and thermodynamic Bethe ansatz, Commun. Math. Phys. 177 (1996) 381 [hep-th/9412229] [INSPIRE].

[17] D. Ridout and J. Teschner, Integrability of a family of quantum field theories related to $\sigma$-models, Nucl. Phys. B 853 (2011) 327 [arXiv:1102.5716] [InSPIRE].

[18] F. Delduc, M. Magro and B. Vicedo, Alleviating the non-ultralocality of coset $\sigma$-models through a generalized Faddeev-Reshetikhin procedure, JHEP 08 (2012) 019 [arXiv: 1204.0766] [INSPIRE]. 
[19] A. Melikyan and G. Weber, On the quantization of continuous non-ultralocal integrable systems, Nucl. Phys. B 913 (2016) 716 [arXiv:1611.02622] [INSPIRE].

[20] F. Delduc, M. Magro and B. Vicedo, Alleviating the non-ultralocality of the $A d S_{5} \times S^{5}$ superstring, JHEP 10 (2012) 061 [arXiv:1206.6050] [INSPIRE].

[21] F. Delduc, M. Magro and B. Vicedo, A lattice Poisson algebra for the Pohlmeyer reduction of the $A d S_{5} \times S^{5}$ superstring, Phys. Lett. B 713 (2012) 347 [arXiv:1204.2531] [inSPIRE].

[22] B. Vicedo, On integrable field theories as dihedral affine Gaudin models, arXiv:1701.04856 [INSPIRE].

[23] C. Appadu, T.J. Hollowood and D. Price, Quantum Inverse Scattering and the Lambda Deformed Principal Chiral Model, arXiv:1703.06699 [INSPIRE].

[24] T.J. Hollowood, J.L. Miramontes and D.M. Schmidtt, S-Matrices and Quantum Group Symmetry of k-Deformed $\sigma$-models, J. Phys. A 49 (2016) 465201 [arXiv:1506.06601] [INSPIRE].

[25] C. Appadu, T.J. Hollowood, J.L. Miramontes, D. Price and D.M. Schmidtt, String Theory In The Lambda Background: Integrability And Gauge Fixing, to appear.

[26] A. Alekseev, L.D. Faddeev and M. Semenov-Tian-Shansky, Hidden quantum groups inside Kac-Moody algebra, Commun. Math. Phys. 149 (1992) 335 [InSPIRE].

[27] A. Alekseev, L. Faddeev, M. Semenov Tian-Shansky and A. Volkov, The unravelling of the quantum group structure in the WZNW theory, CERN-TH-5981-91 (1991).

[28] F. Falceto and K. Gawędzki, Lattice Wess-Zumino-Witten model and quantum groups, J. Geom. Phys. 11 (1993) 251 [hep-th/9209076] [INSPIRE].

[29] A. Yu. Alekseev, L.D. Faddeev, J. Fröhlich and V. Schomerus, Representation theory of lattice current algebras, Commun. Math. Phys. 191 (1998) 31 [q-alg/9604017] [INSPIRE].

[30] V.V. Fock and A.A. Rosly, Poisson structure on moduli of flat connections on Riemann surfaces and r matrix, Am. Math. Soc. Transl. 191 (1999) 67 [math/9802054] [INSPIRE].

[31] A. Yu. Alekseev, H. Grosse and V. Schomerus, Combinatorial quantization of the Hamiltonian Chern-Simons theory, Commun. Math. Phys. 172 (1995) 317 [hep-th/9403066] [INSPIRE].

[32] A. Yu. Alekseev, H. Grosse and V. Schomerus, Combinatorial quantization of the Hamiltonian Chern-Simons theory. 2., Commun. Math. Phys. 174 (1995) 561 [hep-th/9408097] [INSPIRE].

[33] E. Buffenoir and P. Roche, Two-dimensional lattice gauge theory based on a quantum group, Commun. Math. Phys. 170 (1995) 669 [hep-th/9405126] [INSPIRE].

[34] E. Buffenoir and P. Roche, Link invariants and combinatorial quantization of Hamiltonian Chern Simons theory, Commun. Math. Phys. 181 (1996) 331 [q-alg/9507001] [INSPIRE].

[35] D.M. Schmidtt, Integrable Lambda Models and Chern-Simons Theories II, in progress.

[36] V. Mikhaylov and E. Witten, Branes And Supergroups, Commun. Math. Phys. 340 (2015) 699 [arXiv: 1410.1175] [INSPIRE].

[37] V. Mikhaylov, Analytic Torsion, 3d Mirror Symmetry And Supergroup Chern-Simons Theories, arXiv:1505.03130 [INSPIRE]. 
[38] V. Schomerus, Poisson Structure And Quantization Of Chern-Simons Theory, in Quantization of singular symplectic quotients, N.P. Landsman, M. Pflaum and M. Schlichenmaier eds., Springer, (2001) 283.

[39] S.G. Rajeev and O.T. Turgut, Poisson brackets of Wilson loops and derivations of free algebras, J. Math. Phys. 37 (1996) 637 [hep-th/9508103] [INSPIRE].

[40] A. Yu. Alekseev, Integrability in the Hamiltonian Chern-Simons theory, hep-th/9311074 [INSPIRE].

[41] B. Vicedo, Deformed integrable $\sigma$-models, classical R-matrices and classical exchange algebra on Drinfel'd doubles, J. Phys. A 48 (2015) 355203 [arXiv:1504.06303] [InSPIRE].

[42] M. Grigoriev and A.A. Tseytlin, Pohlmeyer reduction of $A d S_{5} \times S^{5}$ superstring $\sigma$-model, Nucl. Phys. B 800 (2008) 450 [arXiv:0711.0155] [INSPIRE].

[43] J.M. Maillet, New Integrable Canonical Structures in Two-dimensional Models, Nucl. Phys. B 269 (1986) 54 [INSPIRE].

[44] E. Witten, Quantum Field Theory and the Jones Polynomial, Commun. Math. Phys. 121 (1989) 351 [INSPIRE].

[45] S. Elitzur, G.W. Moore, A. Schwimmer and N. Seiberg, Remarks on the Canonical Quantization of the Chern-Simons-Witten Theory, Nucl. Phys. B 326 (1989) 108 [inSPIRE].

[46] G.W. Moore and N. Seiberg, Taming the Conformal Zoo, Phys. Lett. B 220 (1989) 422 [INSPIRE].

[47] W. Ogura, Path Integral Quantization of Chern-Simons Gauge Theory, Phys. Lett. B 229 (1989) 61 [INSPIRE].

[48] E. Guadagnini, M. Martellini and M. Mintchev, Perturbative Aspects of the Chern-Simons Field Theory, Phys. Lett. B 227 (1989) 111 [InSPIRE].

[49] L. Álvarez-Gaumé, J.M.F. Labastida and A.V. Ramallo, A Note on Perturbative Chern-Simons Theory, Nucl. Phys. B 334 (1990) 103 [InSPIRE].

[50] C. Appadu, T.J. Hollowood, J.L. Miramontes, D. Price and D.M. Schmidtt, Giant Magnons of String Theory in the Lambda Background, arXiv:1704.05437 [INSPIRE].

[51] R. Borsato, A.A. Tseytlin and L. Wulff, Supergravity background of $\lambda$-deformed model for $A d S_{2} \times S^{2}$ supercoset, Nucl. Phys. B 905 (2016) 264 [arXiv:1601.08192] [INSPIRE].

[52] Y. Chervonyi and O. Lunin, Supergravity background of the $\lambda$-deformed $A d S_{3} \times S^{3}$ supercoset, Nucl. Phys. B 910 (2016) 685 [arXiv: 1606.00394] [INSPIRE].

[53] R. Borsato and L. Wulff, Target space supergeometry of $\eta$ and $\lambda$-deformed strings, JHEP 10 (2016) 045 [arXiv: 1608.03570] [INSPIRE].

[54] A. Yu. Alekseev and V. Schomerus, D-branes in the WZW model, Phys. Rev. D 60 (1999) 061901 [hep-th/9812193] [INSPIRE].

[55] T.J. Hollowood, J.L. Miramontes and D.M. Schmidtt, The Structure of Non-Abelian Kinks, JHEP 10 (2013) 058 [arXiv: 1306.6651] [INSPIRE].

[56] M.-I. Park, Symmetry algebras in Chern-Simons theories with boundary: Canonical approach, Nucl. Phys. B 544 (1999) 377 [hep-th/9811033] [INSPIRE].

[57] P. Oh and M.-I. Park, Symplectic reduction and symmetry algebra in boundary Chern-Simons theory, Mod. Phys. Lett. A 14 (1999) 231 [hep-th/9805178] [InSPIRE]. 
[58] M. Bañados and I.A. Reyes, A short review on Noether's theorems, gauge symmetries and boundary terms, Int. J. Mod. Phys. D 25 (2016) 1630021 [arXiv:1601.03616] [InSPIRE].

[59] T. Regge and C. Teitelboim, Role of Surface Integrals in the Hamiltonian Formulation of General Relativity, Annals Phys. 88 (1974) 286 [INSPIRE].

[60] M. Magro, The Classical Exchange Algebra of AdS $S_{5} \times S^{5}$, JHEP 01 (2009) 021 [arXiv: 0810.4136] [INSPIRE].

[61] B. Vicedo, Hamiltonian dynamics and the hidden symmetries of the $A d S_{5} \times S^{5}$ superstring, JHEP 01 (2010) 102 [arXiv:0910.0221] [InSPIRE].

[62] O. Babelon and D. Bernard, Dressing symmetries, Commun. Math. Phys. 149 (1992) 279 [hep-th/9111036] [INSPIRE].

[63] O. Babelon, D. Bernard and M. Talon, Introduction to Classical Integrable Systems, Cambridge University Press, Cambridge, U.K. (2003).

[64] F. Delduc, T. Kameyama, M. Magro and B. Vicedo, Affine q-deformed symmetry and the classical Yang-Baxter $\sigma$-model, JHEP 03 (2017) 126 [arXiv:1701.03691] [INSPIRE].

[65] E. Witten, Integrable Lattice Models From Gauge Theory, arXiv:1611.00592 [InSPIRE].

[66] K. Costello, Supersymmetric gauge theory and the Yangian, arXiv:1303.2632 [INSPIRE].

[67] K. Costello, Integrable lattice models from four-dimensional field theories, Proc. Symp. Pure Math. 88 (2014) 3 [arXiv: 1308.0370] [INSPIRE].

[68] P. Ševera, Poisson-Lie T-duality as a boundary phenomenon of Chern-Simons theory, JHEP 05 (2016) 044 [arXiv: 1602.05126] [INSPIRE]. 